\title{
Acute Hepatic Failure Caused by Hepatitis B Virus Reactivation in Patients Receiving Immunomodulatory Agents for Autoimmune Diseases
}

\author{
Sung-Yu HSU, ${ }^{1}$ Cheng-Maw HO, ${ }^{1,2}$ Po-Huang LEE, ${ }^{1,2}$ Hong-Shiee LAI, ${ }^{1,2}$ Rey-Heng HU ${ }^{1}$ \\ ${ }^{1}$ Department of Surgery, National Taiwan University Hospital, Taipei, Taiwan \\ ${ }^{2}$ Graduate Institute of Clinical Medicine, College of Medicine, National Taiwan University, Taipei, Taiwan
}

\begin{abstract}
Objectives: The increasing use of novel immunomodulators, such as rituximab, leflunomide and adalimumab in the treatment of autoimmune diseases may put hepatitis B virus (HBV) carriers at increased risk for reactivation and consequent hepatic failure.

Patients and methods: We assessed 164 patients newly registered as liver transplant candidates at the National Taiwan University Hospital in 2010. Four patients (2.4\%) also had underlying autoimmune disease and received immunomodulatory therapy which resulted in HBV reactivation and acute liver failure, which manifested as progressive hyperbilirubinemia and coagulopathy. The median time of survival of these patients was estimated using the Kaplan-Meier method.

Results: All four patients were previously healthy HBV carriers with e antigen seroconversion. The mean peak alanine aminotransferase level was $3194.5 \pm 937.7$ U/L. Entecavir was administered for an average of $39.8 \pm 10.5$ days after the onset of the symptomatic hepatic dysfunction. Three patients who were experienced complications such as hepatic encephalopathy died. Only one patient survived liver failure. The median survival time after HBV reactivation and acute liver failure was 74.0 days ( $95 \%$ confidence interval, 31.9 to 116.1 days). The cumulative survival rate after $\mathrm{HBV}$ reactivation within three months was $25 \%$.

Conclusion: Prophylactic anti-HBV medication is recommended for HBV carriers with autoimmune diseases who are prescribed immunomodulatory agents for preventing catastrophic consequences such as acute liver failure caused by HBV reactivation.

Key words: Acute liver failure; autoimmune disease; Hepatitis B virus reactivation; immunomodulatory therapy.
\end{abstract}

Hepatitis $\mathrm{B}$ virus (HBV) infection is the most common chronic viral liver infection and is the leading cause of cirrhosis and hepatocellular carcinoma in Taiwan. ${ }^{1}$ Reactivation of chronic hepatitis $\mathrm{B}$ infection in patients receiving cytotoxic chemotherapy for malignancy is well documented. ${ }^{2-4}$ Patients who are HBsAgpositive/HBsAb-negative or HBsAg-negative/ $\mathrm{HBcAb}$-positive with HBV DNA-positive serum (occult HBV infection) prior to chemotherapy are at a high risk for $\mathrm{HBV}$ reactivation. ${ }^{5}$ Reactivation of $\mathrm{HBV}$ has also been recognized in patients with rheumatic diseases who receive new immunomodulators, such as tumor necrosis factor-alpha (TNF- $\alpha)$ inhibitors and anti-B cell therapy; this reactivation is a frequently reported complication of considerable clinical importance..$^{6-8}$ Unless accounted for appropriately, these novel therapies may increase the risk for $\mathrm{HBV}$ reactivation and subsequently result in acute liver failure in HBV carriers.

Prophylactic use of nucleoside/nucleotide analogs prior to chemotherapy and their continued use until reconstitution of host immunity remains the mainstay of effective $\mathrm{HBV}$ reactivation prevention. ${ }^{2}$ However, few studies have addressed the issue of prophylactic antiviral therapy in HBV carriers receiving immunomodulatory therapy. 
Herein, we report a series of patients with underlying autoimmune disease who received immunomodulatory therapy and subsequently developed HBV reactivation and acute liver failure.

\section{PATIENTS AND METHODS}

Hepatitis was defined as a three-fold or greater increase in the levels of alanine aminotransferase (ALT) that exceeded the upper limit of the normal level (44 U/L) or an absolute increase to $>100 \mathrm{U} / \mathrm{L}$. Hepatitis B virus reactivation was defined as a change to $\mathrm{HBsAg}$ positive, serum HBV DNA level $>10^{5}$ copies $/ \mathrm{mL}(20,000 \mathrm{IU} / \mathrm{mL})$, or a 10 -fold rise in HBV DNA. ${ }^{9}$ Acute liver failure refers to the rapid development of acute severe liver injury with impaired synthetic function [usually an international normalized ratio (INR) 21.5 ] and encephalopathy in a person with a previously normal liver or a well-compensated liver disease. ${ }^{10,11}$

Among the 164 patients who were newly registered as liver transplant candidates at the National Taiwan University Hospital in 2010, four (2.4\%) had underlying rheumatologic conditions and had received immunosuppressive agents that had resulted in acute liver failure because of $\mathrm{HBV}$ reactivation. All four patients who were candidates for liver transplantation had undergone a thorough clinical evaluation, including radiographic imaging, blood tests for evaluating immunity and possible infections, blood typing and cross-matching, and psychiatric evaluation. The clinical characteristics and courses were retrospectively collected by chart review. The median time of survival with 95\% confidence interval was calculated using the Kaplan-Meier method with the Statistical Package for Social Sciences (SPSS ${ }^{\circledR}$ ) version 13.0 software (SPSS Inc., Chicago, Illinois, USA).

\section{RESULTS}

All patients were previously healthy HBV carriers with $e$ antigen seroconversion. Individual case details are described below.

Case 1- A 54-year-old man with a history of chronic hepatitis $\mathrm{B}$ since childhood without regular follow-up and a four-year history of ankylosing spondylitis initially presented with numbness and pain in his right arm and arthralgia in his right first metacarpophalangeal and left radiocarpal joints. He also presented with morning stiffness, which improved with activity, and limited chest expansion. However, the patient did not experience low back pain or restricted movement of the lumbar spine. Blood test results showed that he was positive for antinuclear antibody (ANA) (1:40, homogeneous) and had an elevated erythrocyte sedimentation rate $(70 \mathrm{~mm})$ and $\mathrm{C}$-reactive protein $(\mathrm{CRP})$ level (3.84 mg/dl). Levels of immunoglobulin A (IgA) (450 mg/dl), IgG (1420 mg/dl), and rheumatoid factor $(\mathrm{RF}<20 \mathrm{IU} / \mathrm{ml})$ were within normal limits.

Sulfasalazine was given initially and then gradually followed with leflunomide, hydroxychloroquine, etanercept, and azathioprine. Polyarthralgia and limited range of motion remained despite treatment. Furthermore, he could not fully flex or extend his fingers or fully flex hip and knee joints, and the external rotation of both humeroglenoid joints was limited. He then received three courses of rituximab (500 mg bi-weekly, two doses per course) in January 2009, March 2010, and July 2010, with methylprednisolone (80 $\mathrm{mg}$ ) as premedication. He later received adalimumab (40 mg bi-weekly for one month) and underwent regular follow-up. His clinical condition improved during the follow-up period.

Four months later, liver enzyme levels (ALT, $467 \mathrm{U} / \mathrm{L}$ ) were elevated, and he complained of dizziness, nausea, poor appetite, and abdominal discomfort for one month. Furthermore, he experienced progressive jaundice and his urine was tea-colored for 10 days. Laboratory data confirmed deteriorating liver function, and the virology surveys revealed high-reactive titers of $\mathrm{HBsAg}(>250.0)$, reactive $\mathrm{HBeAg}$, and anti-HBe. Serology surveys for autoimmune hepatitis $(\mathrm{AIH})$ were all negative. He was given entecavir, admitted to our hospital, and evaluated for liver transplantation. He then rapidly developed grade 1-2 hepatic encephalopathy with coagulopathy (INR $>10$ ), for which he received plasmapheresis. Despite this, he developed hypotension, brain edema, and bacteremia, and he died one month after admission due to multiorgan failure.

Case 2- A 60-year-old man who was an HBV carrier and had a history of hypertension, 
was diagnosed with early rheumatoid arthritis, and initially presented with ankle swelling and polyarthralgia. Laboratory tests indicated elevated $\mathrm{RF}(71.2 \mathrm{IU} / \mathrm{ml})$, erythrocyte sedimentation rate (66 mm), and CRP level (3.22 mg/dl). He was stable under treatment with hydroxychloroquine and methotrexate until six months after presentation, at which when he experienced general malaise, poor appetite, dizziness, dry mouth, yellowish skin color, tea-colored urine, and clay-colored stools for one week. Additionally, his body weight had decreased from $67 \mathrm{~kg}$ to $59 \mathrm{~kg}$ in two weeks. He had a significant history of alcohol abuse ( 1 bottle of Chinese wine every day for over 30 years), but had discontinued alcohol consumption since 10 days because of his condition. He was admitted for the diagnosis of acute liver failure caused by exacerbation of hepatitis $\mathrm{B}$ as evidenced by the elevation of liver enzymes (aspartate aminotransferase, 2170 U/L; ALT, 2663 U/L), hyperbilirubinemia, coagulopathy, high reactive HBsAg (>250 IU/mL), HBV DNA level $\left(8.8 \times 10^{8} \mathrm{IU} / \mathrm{ml}\right)$, and negative serological survey for AIH. Liver transplantation was evaluated. Grade 2 hepatic encephalopathy developed immediately, with elevated ammonia level, prolonged INR, progressive jaundice, fever, and oxygen desaturation. He received hemodialysis for hyperammonemia and intermittent seizures occurred. Refractory shock developed shortly thereafter, and he was discharged despite his condition.

Case 3- A 53-year-old woman, with an unknown HBV status, who had been receiving hydroxychloroquine treatment for antiphospholipid syndrome for the past one year, presented with feet swelling, bilateral knee arthralgia, leukocytosis (white blood cell count $13,980 / \mathrm{mL}$ ), elevated CRP level (16.8 mg/dl), IgG (2100 mg/dl), IgA (351 mg/dl), and C4 (61 mg/dl) levels, positive ANA (1:40 speckled), positive anti-cardiolipin antibody (ACA), and negative treponema pallidum hemagglutination assay (1:80). Her condition improved with hydroxychloroquine treatment (CRP level, $5.2 \mathrm{mg} / \mathrm{dl}$ ). She was admitted for anti-phospholipid syndrome-related pneumonitis with symptoms of chest pain and dyspnea for six days. Laboratory studies revealed leukocytosis (white blood cell count, $46,540 / \mathrm{mL}$ ), negative for ANA, elevated C3 level (146 mg/dl), normal C4 level $(58.8 \mathrm{mg} / \mathrm{dl})$, an anti-DNA antibody level of 36.7 , IgG ACA of 23.95, IgM ACA of 9.58, negative for perinuclear $\mathrm{p}$-anti nuclear cytoplasmic antibody, and positive for cytoplasmic anti nuclear cytoplasmic antibody. Diffuse patchy lesions over the right lung were identified on chest computed tomography. Since low-dose methylprednisolone (60 mg daily for 3 days) improved her condition, she was given a further three-day course of pulse therapy (methylprednisolone $500 \mathrm{mg}$ daily) and was discharged after two weeks with a tapering dose of oral maintenance prednisolone and hydroxychloroquine.

Three months later, abnormal liver function was noted after pulse therapy during a regular follow-up. Her virology profile showed HBV infection with high viral load (DNA level: $>10^{9} \mathrm{IU} / \mathrm{ml}$ ). Entecavir was prescribed, and her general malaise and nausea had improved, but hyperbilirubinemia (total bilirubin level, $12.06 \mathrm{mg} / \mathrm{dl}$ ) and coagulopathy (INR, 1.89) were noted one week later. She was admitted for liver transplant evaluation, but she immediately developed hepatic encephalopathy. One month later, her condition deteriorated to profound shock, bradycardia, then asystole, and she died.

Case 4- A 48-year-old man, previously a healthy HBV carrier, was diagnosed with RA with the initial presentation of right knee pain and increased RF titer (1:80). He received hydroxychloroquine $(200 \mathrm{mg}$ ) twice daily for two months and then once daily for one month, and his symptoms improved. Three months later, however, elevated liver enzyme and bilirubin levels were noted. The patient had tea-colored urine, jaundice, general malaise, nausea, and vomiting. He denied history of alcohol consumption, herbal or other medication, travel, insect bites, or animal contact. His HBV virology profile and DNA levels are shown in Table 1 . He was prescribed lamivudine for drugrelated hepatitis $\mathrm{B}$ acute exacerbation. However, his condition rapidly deteriorated to hepatic failure with encephalopathy. Lamivudine was replaced with entecavir, and he was listed for liver transplantation. The liver decompensation resolved slowly over the course of two months.

Detailed administration of the immunomodulatory agents for all the four patients are listed in the footnote of Table 1. 


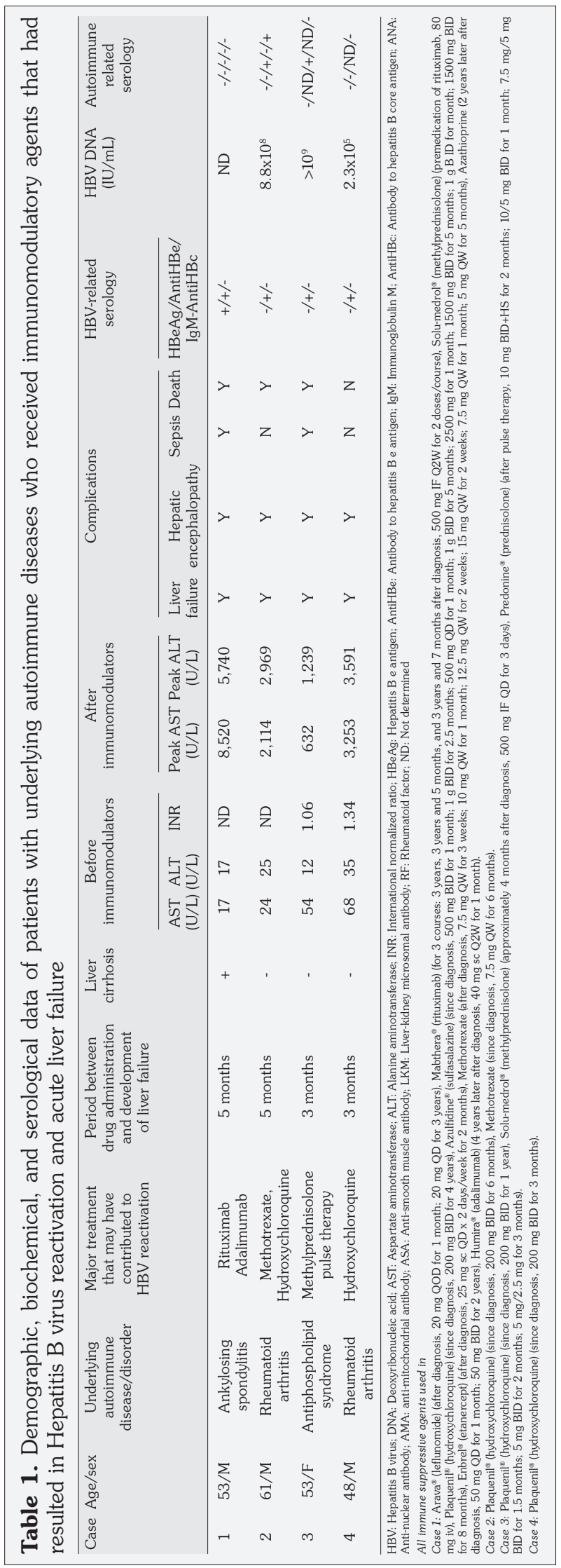

Demographic, biochemical, and serological data of the patients who had underlying autoimmune disease and received immunomodulatory therapy, resulting in HBV reactivation and acute liver failure are summarized in Table 1. Mean peak ALT levels for all four patients was $3194.5 \pm 937.7 \mathrm{U} / \mathrm{L}$. Entecavir was administered since an average of $39.8 \pm 10.5$ days after the start of the symptomatic hepatic dysfunction. Three patients developed complications of hepatic encephalopathy and died. Only one patient (Case 4) survived the episode of acute liver failure. The median time of survival after $\mathrm{HBV}$ reactivation and acute liver failure was 74.0 days $(95 \%$ confidence interval, 31.9-116.1 days). The survival rate after HBV reactivation was approximately 25\% within three months.

\section{DISCUSSION}

Reactivation of HBV occurs when the balance between host immunity and HBV replication is altered. In $\mathrm{HBsAg-positive} \mathrm{patients,} \mathrm{HBV}$ reactivation is a frequent complication, accounting for $21-53 \%$ of $\mathrm{HBV}$ carriers who receive cytotoxic chemotherapy agents for cancer, anti-rejection drugs for transplantation, or corticosteroid. ${ }^{9}$ All of these agents suppress host immunity and facilitate viral replication. Immunologic rebound, which often occurs after sudden withdrawal of these medications, can result in life-threatening consequences in $\mathrm{HBV}$ patients, unless recognized and treated in time with antiviral drugs. ${ }^{6}$ In theory, the more potent the immunosuppressant, the greater the extent of viral replication and therefore, the greater the clinical repercussions of sudden withdrawal. Other risk factors for reactivation include young age, male sex, $\mathrm{HBeAg}$ positivity, a diagnosis of lymphoma or breast cancer, high pretreatment HBV DNA level, prevalence of male homosexuality, human immunodeficiency virus infection, other concurrent bacterial infection or surgery, emotional or physical stress, and pregnancy. ${ }^{6}$ Among these risk factors, HBV DNA level is the most significant predictor of reactivation. ${ }^{5,6}$

Some studies have discussed the relationship between steroid use and HBV replication. 
The glucocorticoid responsive element that is produced by HBV stimulates viral replication and transcriptional activity in host cells. ${ }^{6,12}$ The mechanism of HBV reactivation, which involves viral activation followed by immunologic rebound in response to increased viral antigen expression after the withdrawal of corticosteroid-based immunosuppression, is the same as that observed in cancer chemotherapy. ${ }^{6}$ Newer biological immune modifiers, including rituximab, infliximab, and other anti-TNF- $\alpha$ inhibitors, are more likely to cause reactivation of HBV replication. ${ }^{13}$ However, fewer studies have reported on the mechanism of $\mathrm{HBV}$ reactivation following the use of immunoregulatory drugs such as leflunomide and hydroxychloroquine and the specific risk of reactivation for each regimen is unknown.

Tumor necrosis factor-alpha suppresses HBV replication and plays an important role in eradicating HBV by stimulating an HBV-specific cytotoxic T-cell response. One study proposed that screening for $\mathrm{HBsAg}$ and $\mathrm{HBcAb}$, along with antiviral prophylaxis, might effectively reduce $\mathrm{HBV}$ reactivation in $\mathrm{HBsAg}$-positive RA patients receiving TNF- $\alpha$ inhibitors. In this study, an increase of approximately 47 -fold to 228,505 -fold in viral load was observed during $\mathrm{HBV}$ reactivation compared to that at baseline, and HBV-related hepatitis completely resolved in all patients after lamivudine treatment. ${ }^{9}$ Therefore, we suggest mandatory screening for possible HBV infection before using similar types of immunomodulators, and we further suggest that prophylactic antiviral treatment is necessary for all high-risk patients. Importantly, patients with RA as well as those with other autoimmune diseases should receive prophylactic antiviral treatment in HBV endemic areas, in particular.

When the diagnosis of an acute HBV flareup is delayed until symptoms appear or major biochemical abnormalities occur, it is often too late for antiviral treatment. The antiviral treatment should be started immediately after HBV reactivation is recognized. Patients with underlying cirrhosis may rapidly develop liver failure if proper treatment is not administered, and mortality in such cases ranges from $4 \%$ to $41 \%{ }^{14}$ Despite lamivudine treatment, mortality may be high at $40 \%$, if severe hepatic injury is present. ${ }^{14}$
Therefore, early recognition and prompt antiviral treatment are important for reducing morbidity and mortality. Furthermore, because HBV DNA levels rise prior to ALT level elevation, it is strongly recommended that lamivudine should be administered once an increase in HBV DNA is detected before the appearance of clinical manifestation. Periodic HBV DNA testing may be also indicated.

The use of lamivudine for prophylaxis or for treating reactivated $\mathrm{HBV}$ in the setting of chemotherapy and transplantation has been well studied. Other types of nucleoside analogs, such as entecavir and adefovir, have also been suggested for patients with a prolonged course of chemotherapy or immunosuppressant treatment, because of the relatively high occurrence of lamivudine resistance caused by mutations in HBV (approximately $15 \%$ of cases per year). ${ }^{5}$ However, similar data on populations with autoimmune disorders does not exist. In addition to antiviral treatment, liver transplantation should be considered with high urgency if the hepatic condition deteriorates or there is a trend toward liver decompensation.

In this study, we also found poor outcomes in patients with $\mathrm{HBV}$ infection or autoimmune diseases who were receiving immunoregulatory therapy because of liver failure caused by HBV reactivation. Although in this case series, we reported patients on different drugs and disease types, they all experienced HBV-related acute liver failure secondary to immunomodulatory agents for rheumatologic disorders. This suggests that immune perturbation in HBV carriers under these conditions predisposes patients to a high risk of catastrophic consequences (liver failure or mortality).

All four patients received pre-liver transplant evaluation, including serology to evaluate the status of their rheumatic disease (Table 1) and psychological and social status. One of our patients (Case 4) suffered from hepatic failure after the administration of hydroxychloroquine; this has not been reported previously. We found that immunomodulatory therapy might also result in HBV flare-up similar to that observed after chemotherapy or immunosuppressant therapy, and that increased awareness of this phenomena is needed. While routine 
screening for $\mathrm{HBV}$ in endemic areas prior to chemotherapy and subsequent prophylactic use of anti-HBV therapy for high-risk patients has been advocated, few studies have discussed the importance of applying this concept to the rheumatologic field. This is increasingly important in rheumatology as newer immunomodulatory therapeutic agents directed at immune cell depletion or with anti-cytokine effects are introduced.

In high-risk patients such as HBV carriers and patients with occult HBV infection ( $\mathrm{HBsAg}-$ negative/HBcAb-positive with detectable $\mathrm{HBV}$ DNA), the use of prophylactic antiviral agents and close monitoring of liver function and $\mathrm{HBV}$ DNA titer may be indicated in HBV endemic areas, in particular. We suggest the same policy as with chemotherapy to avoid the irreversible adverse outcomes of treatment and liver failure.

Furthermore, there are some limitations in our study. First, this was a retrospective, cross-sectional, observational study, and the sample size was small. Survival analysis of four patients with three patients dead (censored) is sufficient to perform Kaplan-Meier analysis for an observational study without comparison. It is, however, difficult to reach a statistical conclusion for a small sample size of four even if we compared the results of the other group using the log-rank test of the Kaplan-Meier method. Instead, we only demonstrated a high mortality rate among these patients. More detailed data involving changes in the HBV DNA titer after the use of immunoregulators were not examined regularly. Currently, no study has shown that patients with autoimmune diseases have a high incidence of $\mathrm{HBV}$ infection. However, several studies have suggested that HBV infection may result in some autoimmune diseases such as glomerulonephritis and polyarteritis nodosa. ${ }^{15}$ As newer, more potent immunomodulatory agents are used for rheumatic diseases, these issues will become increasingly important. Future prospective investigations with larger populations are needed.

Mortality in cases of HBV reactivation can be avoided in most patients. We demonstrate that for patients with autoimmune diseases in HBV endemic areas, regular HBV screening and prophylactic use of nucleoside/nucleotide analogs are recommended prior to immunomodulatory therapy and their continuation until reconstitution of host immunity; this will ensure effective prevention of HBV reactivation. More prospective studies are needed to clarify the exact mechanisms of $\mathrm{HBV}$ reactivation after immunomodulatory regimens, and to determine the frequency of HBV DNA titer testing in patients with autoimmune diseases receiving immunoregulatory therapy.

In conclusion, we report four cases of acute hepatic failure, which developed in patients who had received immunosuppressive agents for their rheumatologic conditions. Three of the four patients died due to hepatic failure. We suggest regular HBV screening in HBV-endemic areas, and the use of prophylactic anti-HBV medications for these patients, which is similar to the recommendations for patients receiving chemotherapy.

\section{Declaration of conflicting interests}

The authors declared no conflicts of interest with respect to the authorship and/or publication of this article.

\section{Funding}

The authors received no financial support for the research and/or authorship of this article.

\section{REFERENCES}

1. Yu MW, Hsu FC, Sheen IS, Chu CM, Lin DY, Chen CJ, et al. Prospective study of hepatocellular carcinoma and liver cirrhosis in asymptomatic chronic hepatitis B virus carriers. Am J Epidemiol 1997;145:1039-47.

2. Liu CJ, Chen PJ, Chen DS, Kao JH. Hepatitis $B$ virus reactivation in patients receiving cancer chemotherapy: natural history, pathogenesis, and management. Hepatol Int 2013;7:316-26

3. Lubel JS, Angus PW. Hepatitis B reactivation in patients receiving cytotoxic chemotherapy: diagnosis and management. J Gastroenterol Hepatol 2010;25:864-71.

4. Keam B, Lee JH, Im SA, Yoon JH. Why, when, and how to prevent hepatitis $B$ virus reactivation in cancer patients undergoing chemotherapy. J Natl Compr Canc Netw 2011;9:465-77.

5. Tsai FC, Hsieh SC, Chen DS, Sheu JC, Chen CH. Reactivation of hepatitis $\mathrm{B}$ virus in rheumatologic patients receiving immunosuppressive agents. Dig Dis Sci 2006;51:1627-32. 
6. Perrillo RP. Acute flares in chronic hepatitis B: the natural and unnatural history of an immunologically mediated liver disease. Gastroenterology 2001;120:1009-22.

7. Vento S, Cainelli F, Longhi MS. Reactivation of replication of hepatitis $B$ and $C$ viruses after immunosuppressive therapy: an unresolved issue. Lancet Oncol 2002;3:333-40.

8. Calabrese LH, Zein NN, Vassilopoulos D. Hepatitis B virus (HBV) reactivation with immunosuppressive therapy in rheumatic diseases: assessment and preventive strategies. Ann Rheum Dis 2006;65:983-9.

9. Lan JL, Chen YM, Hsieh TY, Chen YH, Hsieh CW, Chen DY, et al. Kinetics of viral loads and risk of hepatitis $\mathrm{B}$ virus reactivation in hepatitis $\mathrm{B}$ core antibody-positive rheumatoid arthritis patients undergoing anti-tumour necrosis factor alpha therapy. Ann Rheum Dis 2011;70:1719-25.

10. Trey C, Davidson CS. The management of fulminant hepatic failure. In: Popper H, Schaffner F, editors. Progress in liver diseases. Vol. III. New York: Grune \& Stratton; 1970. p. 282-98.

11. Polson J, Lee WM; American Association for the Study of Liver Disease. AASLD position paper: the management of acute liver failure. Hepatology 2005:41:1179-97.

12. Hsieh KP, Huang IW, Hsu CY, Tsai PC. Immunosuppressive therapy in chronic hepatitis $\mathrm{B}$ induced fulminant hepatic failure. J Taiwan Pharm 2010;26:3-9.

13. Lok AS, McMahon BJ. Chronic hepatitis B. Hepatology 2007;45:507-39.

14. Lalazar G, Rund D, Shouval D. Screening, prevention and treatment of viral hepatitis $B$ reactivation in patients with haematological malignancies. $\mathrm{Br} \mathrm{J}$ Haematol 2007;136:699-712.

15. Maya R, Gershwin ME, Shoenfeld Y. Hepatitis B virus (HBV) and autoimmune disease. Clin Rev Allergy Immunol 2008;34:85-102. 